\title{
A THEOREM OF ARCHIMEDES ABOUT SPHERES AND CYLINDERS AND TWO-POINT HOMOGENEOUS SPACES
}

\author{
M. DJORIĆ AND L. VANHECKE
}

\begin{abstract}
Starting from the well-known classical theorem of Archimedes about the volumes of spheres and circumscribing cylinders in three-dimensional Euclidean space, one considers circumscribing tubes of small geodesic spheres in general Riemannian manifolds and one derives new characterisations of two-point homogeneous spaces from it.
\end{abstract}

\section{INTRODUCTION}

It is well-known that in accordance with the expressed desire of Archimedes his tomb was marked by a sphere inscribed in a cylinder. The discovery of the relation between the area and volume of a sphere and a circumscribing cylinder was regarded by him as his most valuable achievement. Archimedes discovered that the ratios between. these volumes are constant in three-dimensional Euclidean space but, of course, this can be extended to $n$-dimensional Euclidean spaces although the values of these ratios depend on the dimension $n$.

The main purpose of this note is to generalise this situation. We will define circumscribing tubes for small geodesic spheres in arbitrary $n$-dimensional Riemannian spaces and study the ratios between the volumes of the geodesic spheres and balls and the circumscribing tubes.

A first result is that the theorem of Archimedes, mentioned above, is a very important one for Euclidean geometry. Indeed, we will show that conversely, if the ratios of the volumes are constant for all small spheres and all circumscribing tubes, then the Riemannian manifold is locally flat. Hence, the property of Archimedes is characteristic for locally Euclidean geometry.

Secondly, we consider similar ratios for the other two-point homogeneous spaces, except the Cayley plane, and we prove that they may also be characterised locally by these ratios. Recall that a two-point homogeneous space is a Riemannian manifold such that its isometry group acts transitively on pairs of equidistant points. This class of spaces is formed by the Euclidean spaces and the rank one symmetric spaces.

Received 20 April 1990

Copyright Clearance Centre, Inc. Serial-fee code: $0004-9729 / 91 \$ \$ 2.00+0.00$. 
Finally, we consider several other relations between the different volumes of geodesic spheres, geodesic balls, circumscribing tubes and geodesic disks and we derive other local characterisations of two-point homogeneous spaces. Several of these relations are also direct generalisations of classical results in Euclidean geometry or are related to some isoperimetric inequalities.

\section{Preliminaries}

We start with some introductory material that will be needed to prove the theorems. For more details we refer to $[7,14]$.

Let $(M, g)$ be an $n$-dimensional $C^{\infty}$ Riemannian manifold which, in the rest of the paper, will be supposed to be connected. Denote by $\nabla$ its Levi Civita connection and by $R$ the associated Riemann curvature tensor defined by

$$
R_{X Y}=\nabla_{[X, Y]}-\left[\nabla_{X}, \nabla_{Y}\right]
$$

for all tangent vector fields $X, Y$. Further, $\rho$ denotes the Ricci tensor, $\tau$ the scalar curvature and $\Delta$ the Laplacian.

Next, let $m \in M$ and let $G_{m}(r)$ denote the geodesic sphere with centre $m$ and radius $r$. We will always suppose that $r$ is sufficiently small in order to have a diffeomorphic exponential map $\exp _{m}$ at $m$. Further, let $S_{m}(r)$ denote the $(n-1)$-dimensional volume of $G_{m}(r)$ and $V_{m}(r)$ the $n$-dimensional volume of the corresponding geodesic ball. Then we have

$$
V_{m}(r)=\int_{0}^{r} S_{m}(t) d t
$$

Some of the coefficients in the Taylor expansions for $S_{m}(r)$ and $V_{m}(r)$ have been computed in $[2,3,14]$. More specifically, we have

$$
\begin{aligned}
& S_{m}(r)=n c_{n} r^{n-1}\left\{1+\frac{1}{6 n} A r^{2}+\frac{1}{360 n(n+2)} B r^{4}+0\left(r^{6}\right)\right\}(m), \\
& V_{m}(r)=c_{n} r^{n}\left\{1+\frac{1}{6(n+2)} A r^{2}+\frac{1}{360(n+2)(n+4)} B r^{4}+0\left(r^{6}\right)\right\}(m)
\end{aligned}
$$

where

$$
A=-\tau, \quad B=-3\|R\|^{2}+8\|\rho\|^{2}+5 \tau^{2}-18 \Delta \tau
$$

Here $c_{n}$ denotes the volume of a unit ball in Euclidean $n$-space, that is

$$
c_{n}=\frac{\pi^{n / 2}}{\left(\frac{n}{2}\right) !}=\frac{\pi^{n / 2}}{\Gamma\left(\frac{n}{2}+1\right)}
$$


These expressions may be derived by using a normal coordinate system $[2,3]$ or with the use of Jacobi vector fields [14].

Now, let $\sigma:[a, b] \rightarrow(M, g)$ be a smooth embedded geodesic through $m$ and denote by $U_{\sigma}(r)$ the tubular neighbourhood of radius $r$ about $\sigma$, that is

$$
\begin{aligned}
U_{\sigma}(r)= & \{p \in M \mid \text { there exits a geodesic } \gamma \text { of } M \text { through } p \text { cutting } \\
& \sigma \text { orthogonally and with length } L(\gamma) \leqslant r\} .
\end{aligned}
$$

We always suppose that the radius $r$ is smaller than the distance from $\sigma$ to its nearest focal point. The $n$-dimensional volume of $U_{\sigma}(r)$ will be denoted by $V_{\sigma}(r) . S_{\sigma}(r)$ will denote the $(n-1)$-dimensional volume of the tube $P_{r}$, that is, the set of points of $U_{\sigma}(r)$ which are at distance $r$ from $\sigma$. Then we have again

$$
V_{\sigma}(r)=\int_{0}^{r} S_{\sigma}(s) d s
$$

Taylor series expansions with remainder term have been computed for these volumes in $[5,6,7,14]$ by using Fermi coordinates, Fermi vector fields and Jacobi vector fields. Let $\sigma$ be parametrised by arc length $t$ and denote by $u$ its unit tangent vector field. Further, let $\left\{e_{i}, i=2, \ldots, n\right\}$ be an arbitrary orthonormal basis of $\{u\}^{\perp}(\sigma(t)) \subset T_{\sigma(t)} M$ at $\sigma(t)$. Then we have

$$
V_{\sigma}(r)=c_{n-1} r^{n-1} \int_{a}^{b}\left\{1+\frac{1}{6(n+1)} A_{\sigma} r^{2}+\frac{1}{360(n+1)(n+3)} B_{\sigma} r^{4}+O\left(r^{6}\right)\right\}(\sigma(t)) d t
$$

where

$$
\begin{gathered}
A_{\sigma}=-\left(\tau+\rho_{u u}\right), \\
B_{\sigma}=-3\|R\|^{2}+8\|\rho\|^{2}+5 \tau^{2}-18 \Delta \tau+33 \nabla_{u u}^{2} \tau-9 \Delta \rho_{u u} \\
+10 \tau \rho_{u u}+2 \sum \rho_{u i}^{2}+14 \sum \rho_{i j} R_{u i u j}-6 \sum R_{u i j k}^{2} \\
-21 \nabla_{u u}^{2} \rho_{u u}-3 \rho_{u u}^{2}-10 \sum R_{u i u j}^{2} .
\end{gathered}
$$

Next, let $D_{m}^{x}(r)$ denote the geodesic disk with centre $m$ and radius $r$ which is perpendicular to $x \in T_{m} M$, that is

$$
D_{m}^{x}(r)=\{p \in M \mid d(p, m) \leqslant r\} \cap \exp _{m}\left(\{x\}^{\perp}\right) .
$$

Then we have the following Taylor expansion for the $(n-1)$-dimensional volume $V_{m}^{x}(r)$ of $D_{m}^{x}(r)($ see $[8,9,11])$ :

(9) $V_{m}^{x}(r)=c_{n-1} r^{n-1}\left\{1+\frac{1}{6(n+1)} A_{m}(x) r^{2}+\frac{1}{360(n+1)(n+3)} B_{m}(x) r^{4}+0\left(r^{6}\right)\right\}$ 
where

$$
\begin{gathered}
A_{m}(x)=-\left(\tau-2 \rho_{x x}\right)(m) \\
B_{m}(x)=\left\{-3\|R\|^{2}+8\|\rho\|^{2}+5 \tau^{2}-18 \Delta \tau+24 \sum R_{x i j k}^{2}\right. \\
+20 \sum R_{x i x j}^{2}-8 \sum \rho_{x i}^{2}+12 \rho_{x x}^{2}-16 \sum \rho_{i j} R_{x i x j} \\
\left.-20 \tau \rho_{x x}+36 \sum \nabla_{i i}^{2} \rho_{x x}+18 \nabla_{x x}^{2} \tau\right\}(m)
\end{gathered}
$$

where $\left\{e_{i}, i=1, \ldots, n\right\}$ is an arbitrary orthonormaal basis of $\{x\}^{\perp} \subset T_{m} M$.

Now, we consider the circumscribing tubes and start with the natural definition. Let $m \in M$ and let $G_{m}(r)$ be the geodesic sphere with centre $m$ and radius $r$. Next, let $\sigma:[-r, r] \rightarrow(M, g)$ be a unit speed geodesic through $m=\sigma(0)$. Then, the tube $P_{r}$ about $\sigma$ is by definition the circumscribing tube of the geodesic sphere with $\sigma$ as axial curve. We denote this by $P_{m \sigma}^{c}(r)$. The $n$-dimensional volume of it will be denoted by $V_{\sigma}^{c}(r)$ and the $(n-1)$-dimensional volume by $S_{\sigma}^{c}(r)$. Then, from (5) and (6) we get easily

$$
\begin{aligned}
V_{\sigma}^{c}(r)=c_{n-1} r^{n} & \left\{2+\frac{1}{3(n+1)} A_{\sigma} r^{2}+\frac{1}{18}\left(\frac{1}{10(n+1)(n+3)} B_{\sigma}+\frac{1}{n+1} A_{\sigma}^{\prime \prime}\right) r^{4}\right. \\
+ & \left.0\left(r^{6}\right)\right\}(m) \\
S_{\sigma}^{c}(r)=c_{n-1} r^{n-1} & \left\{2(n-1)+\frac{1}{3} A_{\sigma} r^{2}+\frac{1}{18}\left(\frac{1}{10(n+1)} B_{\sigma}+A_{\sigma}^{\prime \prime}\right) r^{4}\right. \\
+ & \left.0\left(r^{6}\right)\right\}(m)
\end{aligned}
$$

where the prime denotes covariant differentiation along $\sigma$.

We also want to have an expression for the $(n-1)$-dimensional total volume $T S_{\sigma}^{c}(r)$ of the circumscribing tube $P_{m \sigma}^{c}(r)$. This volume is given by the formula

$$
T S_{\sigma}^{c}(r)=S_{\sigma}^{c}(r)+V_{\sigma(r)}^{v}(r)+V_{\sigma(-r)}^{\bar{v}}(r)
$$

where $v=\sigma^{\prime}(r)$ and $\bar{v}=\sigma^{\prime}(-r)$. Therefore, we need expressions for the last two terms in (14). From (9) we obtain

$$
\begin{aligned}
V_{\sigma(r)}^{v}(r)= & c_{n-1} r^{n-1}\left\{1+\frac{1}{6(n+1)} A_{m}(x) r^{2}+\frac{1}{6(n+1)} A_{m}^{\prime}(x) r^{3}\right. \\
& \left.+\frac{1}{12(n+1)}\left[A_{m}^{\prime \prime}(x)+\frac{1}{30(n+3)} B_{m}(x)\right] r^{4}+0\left(r^{5}\right)\right\},
\end{aligned}
$$


and

$$
\begin{aligned}
V_{\sigma(-r)}^{\bar{v}}(r)= & c_{n-1} r^{n-1}\left\{1+\frac{1}{6(n+1)} A_{m}(x) r^{2}-\frac{1}{6(n+1)} A_{m}^{\prime}(x) r^{3}\right. \\
& \left.+\frac{1}{12(n+1)}\left[A_{m}^{\prime \prime}(x)+\frac{1}{30(n+3)} B_{m}(x)\right] r^{4}+0\left(r^{5}\right)\right\}
\end{aligned}
$$

where $x=\sigma^{\prime}(0)$. Hence, (13), (14), (15) and (16) yield

$$
\begin{aligned}
T S_{\sigma}^{c}(r)= & c_{n-1} r^{n-1}\left\{2 n+\frac{1}{3}\left(A_{\sigma}(m)+\frac{1}{n+1} A_{m}(x)\right) r^{2}\right. \\
& +\left(\frac{1}{180(n+1)} B_{\sigma}(m)+\frac{1}{18} A_{\sigma}^{\prime \prime}(x)+\frac{1}{6(n+1)} A_{m}^{\prime \prime}(x)\right. \\
& \left.\left.+\frac{1}{180(n+1)(n+3)} B_{m}(x)\right) r^{4}+0\left(r^{6}\right)\right\} .
\end{aligned}
$$

Later on we shall need more detailed Taylor expansions when $\operatorname{dim} M=2$. In this case, let $K$ denote the Gauss curvature. Then we have [4]

$$
\begin{aligned}
& V_{m}(r)=\pi r^{2}\left\{1-\frac{1}{12} \alpha r^{2}+\frac{1}{720} \beta r^{4}+\frac{1}{161280} \gamma r^{6}+0\left(r^{8}\right)\right\}(m), \\
& S_{m}(r)=2 \pi r\left\{1-\frac{1}{6} \alpha r^{2}+\frac{1}{240} \beta r^{4}+\frac{1}{40320} \gamma r^{6}+0\left(r^{8}\right)\right\}(m)
\end{aligned}
$$

where

$$
\begin{gathered}
\alpha=K, \\
\beta=2 K^{2}-3 \Delta K, \\
\gamma=-8 K^{3}+30\|\nabla K\|^{2}+42 K \Delta K-15 \Delta^{2} K .
\end{gathered}
$$

Further, using the method developed in $[5,6]$ one gets after some straightforward calculation, which we omit:

(23)

$$
\begin{aligned}
V_{\sigma}^{c}(r) & =r^{2}\left\{4-\frac{2}{3} K r^{2}+\frac{1}{90}\left(3 K^{2}-3 \nabla_{v v}^{2} K-10 \nabla_{u u}^{2} K\right) r^{4}\right. \\
& +\frac{1}{1260}\left(-7 \nabla_{u u u v}^{4} K-7 \nabla_{u v v v}^{4} K-\nabla_{v v v v}^{4} K+14 K \nabla_{u u}^{2} K+7 K \nabla_{v v}^{2} K\right. \\
& \left.\left.+14\left(\nabla_{u} K\right)^{2}+4\left(\nabla_{v} K\right)^{2}-K^{3}\right) r^{6}+0\left(r^{8}\right)\right\}(m),
\end{aligned}
$$

(24)

$$
\begin{aligned}
S_{\sigma}^{c}(r) & =r\left\{4-2 K r^{2}+\frac{1}{6}\left(-2 \nabla_{u u}^{2} K-\nabla_{v v}^{2} K+K^{2}\right) r^{4}\right. \\
& +\frac{1}{180}\left(-3 \nabla_{u u u v}^{4} K-5 \nabla_{u u v v}^{4} K+10 K \nabla_{u u}^{2} K+10\left(\nabla_{u} K\right)^{2}-\nabla_{v v v v}^{4} K\right. \\
& \left.\left.+7 K \nabla_{v v}^{2} K+4\left(\nabla_{v} K\right)^{2}-K^{3}\right) r^{0}+0\left(r^{8}\right)\right\}(m)
\end{aligned}
$$


where $(u, v)$ is an orthonormal basis of $T_{m} M$ with $u=\sigma^{\prime}(0)$. Note that in this case

$$
V_{\sigma(r)}^{v}=V_{\sigma(-r)}^{\bar{v}}=2 r
$$

We note that for the two-point homogeneous spaces it is possible to write down the explicit formulas for all the volumes considered above. This has been done in [2, 3, 6, $7,11,13,14]$. Here we do not write down these expressions because we shall not need them in general. The only result we will use follows directly from these expressions or may be derived in a more general way from [10]. We formulate it as follows:

Lemma 1. Let $(M, g)$ be a two-point homogeneous space. Then all the volumes of the geodesic spheres and balls, the circumscribing tubes and the geodesic disks are independent of $m=\sigma(0)$ and $x=\sigma^{\prime}(0)$, that is, they depend only on the radius $r$.

We finish this section by some integration formulas which we shall need in the proof of our theorems. They are derived in $[1,3]$.

LEMMA 2. We have, with $n=\operatorname{dim} M$,

$$
\begin{aligned}
\int_{S^{n-1}(1)} \sum R_{u i u j}^{2}(m) d u & =\frac{1}{n+2} c_{n}\left(\|\rho\|^{2}+\frac{3}{2}\|R\|^{2}\right)(m), \\
\int_{S^{n-1}(1)} \sum R_{u i j k}^{2}(m) d u & =\frac{n-1}{n+2} c_{n}\left(\|R\|^{2}-\frac{2}{n-1}\|\rho\|^{2}\right)(m)
\end{aligned}
$$

where $u$ is a unit vector of $T_{m} M$ and $\left\{e_{i}, i=2, \ldots, n\right\}$ is an arbitrary orthonormal basis of $\{u\}^{\perp} \subset T_{m} M$.

From this we get

Corollary 3. For an n-dimensional two-point homogeneous space we have

$$
\begin{aligned}
& \sum R_{u i u j}^{2}=\frac{1}{n(n+2)}\left(\|\rho\|^{2}+\frac{3}{2}\|R\|^{2}\right) \\
& \sum R_{u i j k}^{2}=\frac{n-1}{n(n+2)}\left(\|R\|^{2}-\frac{2}{n-1}\|\rho\|^{2}\right) .
\end{aligned}
$$

\section{ARCHIMEDES-LIKE THEOREMS AND TWO-POINT HOMOGENEOUS SPACES}

In this section we shall consider direct analogues of the theorem of Archimedes. As concerns circumscribing tubes we may consider the volume-ratios

$$
V_{\sigma}^{c} / V_{m}, \quad S_{\sigma}^{c} / S_{m}, T S_{\sigma}^{c} / S_{m}
$$

From the remark made in Section 2 we may conclude that these ratios depend only on the radius when $(M, g)$ is a two-point homogeneous space. In fact, using the explicit 
expressions for these volumes, one may write down the ratios for each of the two-point homogeneous spaces. Our main purpose is to determine to what extent these explicit expressions determine these spaces up to local isometry. We shall show that they do that completely.

We start with a more general theorem.

THEOREM 4. Let $\left(M_{0}^{n}, g_{0}\right)$ be an n-dimensional two-point homogeneous space and $\left(M^{n}, g\right)$ an $n$-dimensional Riemannian manifold such that for all $m \in M$, all geodesics $\sigma$ through $m$ and all sufficiently small $r$ we have that the ratios

(i) $V_{\sigma}^{c} / V_{m}$, or

(ii) $S_{\sigma}^{c} / S_{m}$, or

(iii) $T S_{\sigma}^{c} / S_{m}$

are equal to the corresponding ratios for $\left(M_{0}^{n}, g_{0}\right)$. Then $(M, g)$ is an Einstein space and moreover,

$$
\tau=\tau_{0}, \quad\|R\|=\left\|R_{0}\right\| .
$$

Proof: We give a proof for the case (i). The proofs for the other cases are similar.

We use (2), (3), (12), (7) and (8) and start by putting

$$
\frac{V_{\sigma}^{c}(r)}{V_{m}(r)}=a \frac{1+\lambda(m) r^{2}+\mu(m) r^{4}+0\left(r^{6}\right)}{1+\xi(m) r^{2}+\eta(m) r^{4}+0\left(r^{6}\right)}
$$

where $a=2 c_{n-1} / c_{n}$. For the model space $\left(M_{0}, g_{0}\right)$ we put

$$
\frac{V_{0}^{c}(r)}{V_{0}(r)}=a \frac{1+\lambda_{0} r^{2}+\mu_{0} r^{4}+0\left(r^{6}\right)}{1+\xi_{0} r^{2}+\eta_{0} r^{4}+0\left(r^{6}\right)}
$$

The equality of the expressions (30) and (31), for all $r$, yields as first necessary condition

$$
\lambda-\xi=\lambda_{0}-\xi_{0}
$$

for all $m \in M$. Hence, and since $\left(M_{0}, g_{0}\right)$ is an Einstein space (that is, $\left.\rho_{0}=\left(\tau_{0} / n\right) g_{0}\right)$, we get

$$
\frac{1}{n+1}\left(\tau+\rho_{u u}\right)-\frac{1}{n+2} \tau=\frac{2}{n(n+2)} \tau_{0}
$$

Since this must be true for any unit vector $u$, we may put $u=e_{i}$ where $\left\{e_{i}, i=\right.$ $1, \ldots, n\}$ is an arbitrary orthonormal basis of $T_{m} M$. Then (32) gives, by summing up,

$$
\tau=\tau_{0}
$$


Substituting this in (32) yields

$$
\rho_{\text {uv }}=\frac{\tau}{n}
$$

which implies $\rho=(\tau / n) g$, that is, $(M, g)$ is an Einstein space. Note that (33) implies that $\tau$ is constant.

Next, we use these results to write down the second necessary condition. This condition becomes

$$
\mu-\eta=\mu_{0}-\eta_{0}
$$

where

$$
\begin{gathered}
360(\eta-\mu)=\frac{1}{(n+2)(n+4)}\left(-3\|R\|^{2}+\frac{5 n+8}{n} \tau^{2}\right) \\
+\frac{1}{(n+1)(n+3)}\left[3\|R\|^{2}+6 \sum R_{u i j k}^{2}+10 \sum R_{u i u j}^{2}-\frac{5 n^{2}+18 n+11}{n^{2}} \tau^{2}\right] .
\end{gathered}
$$

So, we have

$$
+(n+2)(n+4)\left(6 \sum R_{u i j k}^{2}+10 \sum R_{u i u j}^{2}\right)-\frac{1}{n^{2}}\left(20 n^{3}+112 n^{2}+186 n+88\right) \tau^{2} \text {. }
$$

Now, we integrate this over the unit sphere in $T_{m} M$ and use (26) and (27) to get

$$
\begin{gathered}
360(n+1)(n+2)(n+3)(n+4) \int_{S^{n-1}(1)}(\eta-\mu) d u= \\
n c_{n}\left[3(2 n+5)\|R\|^{2}+\frac{6(n-1)(n+4)}{n}\left(\|R\|^{2}-\frac{2}{n-1}\|\rho\|^{2}\right)\right. \\
\left.+\frac{10(n+4)}{n}\left(\|\rho\|^{2}+\frac{3}{2}\|R\|^{2}\right)-\frac{1}{n^{2}}\left(20 n^{3}+112 n^{2}+186 n+88\right) \tau^{2}\right] .
\end{gathered}
$$

Doing this also for the model space, or using (28) and (29), the second condition leads at once to

$$
\|R\|=\left\|R_{0}\right\|,
$$

which proves the required result.

From this theorem we obtain at once the converse of the theorem of Archimedes.

Corollary 5. Let $\left(M^{n}, g\right)$ be a Riemannian manifold such that for all $m \in M$, all geodesics $\sigma$ through $m$ and all sufficiently small $r$,
(i) $V_{\sigma}^{c} / V_{m}$, or
(ii) $S_{\sigma}^{c} / S_{m}$, or
(iii) $T S_{\sigma}^{c} / S_{m}$ 
are constant. Then $(M, g)$ is locally flat.

Proof: The constancy of the expressions is equivalent to the choice of a flat model space. Then Theorem 4 implies at once $R=0$.

This corollary is a special case of the next one.

COROLlary 6. Let $\left(M^{n}, g\right)$ be a Riemannian manifold such that for all $m \in M$, all geodesics $\sigma$ theough $m$ and all sufficiently small $r$,

(i) $V_{\sigma}^{c} / V_{m}$, or

(ii) $S_{\sigma}^{c} / S_{m}$, or

(iii) $T S_{\sigma}^{c} / S_{m}$

are the same as the corresponding ratios for a manifold of constant sectional curvature $\left(M_{0}, g_{0}\right)$. Then $(M, g)$ is locally isometric to $\left(M_{0}, g_{0}\right)$.

Proof: Use Theorem 4 and the fact that a Riemannian manifold $\left(M^{n}, g\right)$ has constant sectional curvature if and only if

$$
\|R\|^{2}=\frac{2}{n(n-1)} \tau^{2}
$$

(see $[1,3,6]$ for a reference).

Similarly we have

Corollary 7. Let $\left(M^{2 n}, g, J\right)$ be a Kähler manifold with complex dimension $n$ such that for all $m \in M$, all geodesics $\sigma$ through $m$ and all sufficiently small $r$,

(i) $V_{\sigma}^{c} / V_{m}$, or

(ii) $S_{\sigma}^{c} / S_{m}$, or

(iii) $T S_{\sigma}^{\mathrm{c}} / S_{m}$

are the same as the corresponding expressions for a Kähler manifold $\left(M_{0}^{2 n}, g_{0}, J_{0}\right)$ of constant holomorphic sectional curvature. Then $(M, g, J)$ is locally isometric to $\left(M_{0}, g_{0}, J_{0}\right)$.

Proof: This follows again from Theorem 4 and the fact that a Kähler manifold $\left(M^{2 n}, g, J\right)$ has constant holomorphic sectional curvature if and only if

$$
\|R\|^{2}=\frac{2}{n(n+1)} \tau^{2}
$$

(see $[1,3,6]$ for a reference).

Finally, we have

COROLlary 8. Let $\left(M^{4 n}, g\right)$ be a Riemannian manifold whose holonomy group is a subgroup of $S p(n) \cdot S p(1)$ and such that for all $m \in M$, all geodesics $\sigma$ through $m$ and all sufficiently small $r$,

(i) $V_{\sigma}^{c} / V_{m}$, or 
(ii) $S_{\sigma}^{c} / S_{m}$, or

(iii) $T S_{\sigma}^{c} / S_{m}$

are the same as the corresponding ratios for a quaternionic space form $\left(M_{0}^{4 n}, g_{0}\right)$. Then $\left(M^{4 n}, g\right)$ is locally isometric to $\left(M_{0}^{4 n}, g_{0}\right)$.

Proof: Use Theorem 4 together with the characterisation property

$$
\|R\|^{2}=\frac{5 n+1}{4 n(n+2)^{2}} \tau^{2}
$$

for a quaternionic space form (see $[1,3,6]$ for a reference).

\section{ANOThER SERIES OF RESUlts}

Two-point homogeneous spaces may be characterised in a similar way by considering other ratios and by using the formulas given in Section 2 . We give some examples and omit the detailed proofs.

EXAmple A. Theorem 4 and its corollaries have analogues when we consider the ratios

$$
V_{\sigma}^{c} / S_{\sigma}, \quad V_{\sigma}^{c} / T S_{\sigma}^{c}, \quad T S_{\sigma}^{c} / S_{\sigma}
$$

which are determined by volumes associated with the circumscribing tubes alone.

EXAmple B. A classical theorem for three-dimensional Euclidean spaces states that the area of a sphere equals four times the area of a disk with the same centre and radius as the sphere, that is,

$$
S_{m}(r)=4 \pi r^{2} .
$$

As a generalisation of this, we may consider the ratio

$$
V_{m}^{z} / S_{m}
$$

and prove the analogues of Theorem 4 and its corollaries.

EXAMPLE C. In two-dimensional Euclidean geometry we have

$$
V_{\sigma}^{c}=\frac{1}{\pi^{2}} S_{m}^{2}
$$

(see [12] for a relation with an isoperimetric inequality). One obtains a generalisation with analogue results as above by considering the ratio

$$
\left(V_{\sigma}^{c}\right)^{n-1} / S_{m}^{n}
$$

on an $n$-dimensional Riemannian manifold. 
EXAMPLE D. Similar results hold when one considers the ratio

$$
\frac{T S_{\sigma}^{c}}{S_{m}}: \frac{V_{\sigma}^{c}}{V_{m}}
$$

for an $n$-dimensional manifold where $n>2$. The situation is different for surfaces. Indeed, it is easy to see, using the explicit expressions, that when the surface is locally flat or has constant Gauss curvature we always have

$$
\frac{T S_{\sigma}^{c}}{S_{m}}=\frac{V_{\sigma}^{c}}{V_{m}}
$$

Explicitly, in the first case we have

$$
S_{m}=2 \pi r, \quad V_{m}=\pi r^{2}, \quad V_{\sigma}^{c}=4 r^{2}, \quad T S_{\sigma}^{c}=8 r
$$

and for a surface of constant curvature $K>0$ one obtains

$$
\begin{aligned}
S_{m}=2 \pi \frac{\sin \sqrt{K} r}{\sqrt{K}}, & V_{m}=\frac{2 \pi}{K}(1-\cos \sqrt{K} r) \\
V_{\sigma}^{c}=4 r \frac{\sin \sqrt{K} r}{\sqrt{K}}, & T S_{\sigma}^{c}=4 r(1+\cos \sqrt{K} r)
\end{aligned}
$$

(For $K<0$ one has similar expressions using hyperbolic functions instead of trigonometric functions.)

Conversely, we have

Theorem 9. Let $(M, g)$ be a two-dimensional Riemannian manifold such that for all $m \in M$, all geodesics through $m$ and all sufficiently small $r$,

$$
\frac{T S_{\sigma}^{c}}{S_{m}}=\frac{V_{\sigma}^{c}}{V_{m}} .
$$

Then $(M, g)$ has constant curvature.

Proof: The proof uses again the Taylor series expansions but now one has to use the expansions (18), (19), (23) and (24) because one needs more terms to conclude. (We delete the straightforward computations.)

EXAMPLE E. The weakest result we have is the one considering the ratio

$$
\left(\frac{V_{\sigma}^{c}}{S_{\sigma}^{c}}\right)^{n-1} /\left(T S_{\sigma}^{c}-S_{\sigma}^{c}\right)
$$

Here we can only formulate similar results as in $\operatorname{Section} 3$ if we suppose that $(M, g)$ is an Einstein space. We are unable to give a proof when one deletes this condition. 


\section{REFERENCES}

[1] B.Y. Chen and L. Vanhecke, 'Differential geometry of geodesic spheres', J. Reine Angew. Math. 325 (1981), 28-67.

[2] A. Gray, 'The volume of a small geodesic ball in a Riemannian manifold', Michigan Math. J. 20 (1973), 329-344.

[3] A. Gray and L. Vanhecke, 'Riemannian geometry as determined by the volumes of small geodesic balls', Acta Math. 142 (1979), 157-198.

[4] A. Gray and L. Vanhecke, 'Oppervlakten van geodetische cirkels op oppervlakken', Med. Konink. Acad. Wetensch. België 42 (1980), 1-17.

[5] A. Gray and L. Vanhecke, 'The volumes of tubes in a Riemannian manifold', Rend. Sem. Mat. Univ. Politec. Torino 39 (1981), 1-50.

[6] A. Gray and L. Vanhecke, 'The volumes of tubes about curves in a Riemannian manifold', Proc. London Math. Soc. 44 (1982), 215-243.

[7] A. Gray, Tubes (Addison-Wesley Publ. Co., Reading, 1989).

[8] O. Kowalski and L. Vanhecke, 'Ball-homogeneous and disk-homogeneous Riemannian manifolds', Math. Z. 180 (1982), 429-444.

[9] O. Kowalski and L. Vanhecke, 'On disk-homogeneous symmetric spaces', Ann. Global Anal. Geom. 1 (1983), 91-104.

[10] O. Kowalski and L. Vanhecke, ' $G$-deformations of curves and volumes of tubes in Riemannian manifolds', Geom. Dedicata 15 (1983), 125-135.

[11] O. Kowalski and L. Vanhecke, 'The volume of geodesic disks in a Riemannian manifold', Czechoslovak Math. J. 35 (1985), 66-77.

[12] E. Lutwak, 'On isoperimetric inequalities related to a problem of Moser', Amer. Math. Monthly 86 (1979), 476-477.

[13] S. Segura Gomis, Teoremas de comparación ligados al problema isoperimétrico, Tesis Doctoral (Departamento de Geometria y Topologia, Universidad de Valencia, Valencia, 1987).

[14] L. Vanhecke, Geometry in Normal and Tubular Neighborhoods, Lecture Notes (Department of Mathematics, University of Leuven, Leuven, 1988). Proc. Workshop on Differential Geometry and Topology (Cala Gonone, Sardinia 1988). (to appear).

Faculty of Mathematics

University of Belgrade

P.B. 550

Studentski trg 16

11000 Belgrade

Yugoslavia
Department of Mathematics

Katholieke Universiteit Leuven

Celestijnenlaan 200B

B-3001 Leuven

Belgium 